Diabetologe 2015 - 11:371-372

DOI 10.1007/s11428-015-1355-4

Online publiziert: 10. Juli 2015

(c) Springer-Verlag Berlin Heidelberg 2015
R.E. Schmieder ${ }^{1} \cdot$ R.G. Bretzel ${ }^{2,3}$

${ }^{1}$ Klinische Forschungsstation CRC, Medizinische Klinik 4, Universitätsklinikum Erlangen

${ }^{2}$ Ehem. Ordinarius und Direktor der Medizinischen Klinik III und Poliklinik, Universitätsklinikum Gießen

${ }^{3}$ Privatpraxis, Gießen

\section{Hypertonie und Diabetes}

Gegenwärtig sind in Deutschland - nach Daten des Robert Koch-Instituts, Berlin, 2012 - mehr als 6 Mio. Menschen von einem Diabetes mellitus betroffen, darunter etwa 5\% vom Typ-1- und 95\% vom Typ-2-Diabetes [Deutsche Diabetes-Gesellschaft (DDG) 2015, www.ddg. info]. Verglichen mit der Prävalenz im Jahre 1998 bedeutet dies eine Steigerung um 38\% in knapp 15 Jahren, und jährlich kommen etwa 270.000 Neuerkrankungen hinzu. Die direkten Kosten für Diabetes pro Jahr liegen schon heute bei $48 \mathrm{Mrd}$. Euro, wobei der Löwenanteil von $80 \%$ für die Behandlung der diabetischen Folgeerkrankungen aufgebracht werden muss: Herzinfarkt/Herzinsuffizienz, Schlaganfall, Nierenerkrankung bis hin zur Nierenersatztherapie, Netzhauterkrankung bis zur Erblindung und diabetisches Fußsyndrom bis hin zu Amputationen.

\section{》) Eine Hypertonie als der weltweit hauptsächliche Risikofaktor kardiovaskulärer Todesfälle ist bei Diabetes häufig}

Nach einer weltweiten Vergleichsstudie stiegen die durch den klassischen kardiovaskulären Risikofaktor Hypertonie bedingten Todesfälle global von 7,3 Mio. im Jahr 1990 binnen 20 Jahren um weitere 2,1 Mio. auf 9,4 Mio. im Jahr 2010 $(+29 \%)$ an [1]. Aus verschiedenen epidemiologischen Studien zusammengefasst sind Todesfälle durch Schlaganfall oder Herzinfarkt zu etwa 50\% bzw. 40\% Folge einer Hypertonie. Pro Zunahme des systolischen/diastolischen Blutdrucks um 20/10 mmHg verdoppelt sich jeweils das kardiovaskuläre Mortalitätsrisiko [2].

Patienten mit einem Diabetes mellitus weisen häufig eine Hypertonie auf, wobei sich Typ-1- und Typ-2-Diabetes wesentlich unterscheiden. Bei Typ-1-Diabetes tritt eine Hypertonie in der Regel erst mit zunehmender Dauer der Erkrankung auf. Gemäß einer deutschen Studie ist nach 10, 20 und 30 Jahren Diabetes mit Prävalenzen von 15, 20 bzw. $40 \%$ zu rechnen [3]. Aus dänischen Untersuchungen wissen wir, dass die Hypertonieprävalenz bei Typ-1-Diabetes weniger an die Jahre der Erkrankung als an das Auftreten einer Mikro- bzw. Makroalbuminurie gekoppelt ist [4]. So lagen die Zahlen für Patienten mit Normalbuminurie bei 19\%, mit Mikroalbuminurie bei $30 \%$, mit Makroalbuminurie bei $65 \%$. Bei Typ-2-Diabetes hingegen wies jeder 2. Patient in der deutschen Studie bereits bei der Erstdiagnose gleichzeitig eine Hypertonie auf, wohl bedingt durch das lange Vorstadium des sog. metabolischen Syndroms. Nach längerer Erkrankungsdauer steigt die Prävalenz auf 80-90\% [3].

Das kardiovaskuläre Risikoprofil des Patienten mit Typ-2-Diabetes wird nicht allein durch die Hypertonie bestimmt. Vielmehr sind häufig neben der diabetischen Stoffwechsellage eine Hyper- oder Dyslipidämie sowie weitere Risikomarker, wie eine Proinflammation und Prokoagulopathie, vorhanden. Es wundert daher nicht, dass Patienten mit Typ-2-Diabetes als kardiovaskuläre Hochrisikogruppe einzustufen sind. Sowohl ihre kardiovaskuläre Morbidität als auch Mortalität sind nach den Daten des deutschen MO-
NICA-Registers ["Multinational monitoring trends and determinants in cardiovascular disease"; Projekt der Weltgesundheitsorganisation (WHO), 1976-2002] bei den Männern 4- bis 5fach, bei den Frauen sogar 6- bis 7fach höher als die altersentsprechender nicht an Diabetes erkrankter Personen [5].

\section{I) Im Kontext Hypertonie und Diabetes werden 5 wichtige Themenkomplexe betrachtet}

Nicht alle Facetten der Hochrisikokonstellation Hypertonie und Diabetes können im Rahmen dieses Schwerpunkthefts abgehandelt werden. Als Herausgeber beschränkten wir uns daher auf 5 Themen und sind uns natürlich dieser subjektiven Auswahl bewusst.

Bönner beschreibt die aktuellen Leitlinien zur Behandlung der arteriellen Hypertonie und die Besonderheiten bei Patienten mit einem Diabetes mellitus.

Savvaidis, Marx und Schütt berichten über die kardialen Aspekte der Hypertonie bei Patienten mit Diabetes. Jahrelang standen dabei das koronare Ereignis/der Herzinfarkt im Vordergrund der Diskussionen. Wir baten die Autoren daher, ihren Beitrag mehr auf die Herzinsuffizienz $\mathrm{zu}$ fokussieren.

Lüders und Schrader widmen sich einem weiteren oft vernachlässigten Thema, dem Schlaganfall als unverändert zweithäufigster Todesursache in unserem Land. Auch in ihrem Artikel werden die diabetesspezifischen Belange herausgestrichen. 
Darf/soll ein Patient mit Hypertonie überhaupt Sport treiben? Ketelhut beantwortet diese Frage kompetent und bringt das Thema auf den Punkt: Sport bei Hypertonie ist sehr effektiv, hat keine Nebenwirkungen und kostet zudem nicht viel!

Wenn die medikamentöse Hypertonietherapie versagt - welche Optionen verbleiben? Ott beschreibt dazu kritisch abwägend die Indikationen und den aktuellen Stand interventioneller Verfahren als Ultima ratio bei schwer einstellbarer Hypertonie.

Wir danken allen Autoren und Koautoren, dass sie trotz ihrer beruflichen Mehrfachbelastungen Zeit fanden, die Artikel zu verfassen und termingerecht zur Publikation einzureichen.

Trotz der Fokussierung in diesem Schwerpunktheft auf die Hypertonie dürfen wir das kardiovaskuläre Gesamtrisikoprofil diabetischer Patienten nicht aus dem Auge verlieren. In vielen Interventionsstudien - wenn zuletzt auch mit durchaus kontroversen Resultaten - zeigte sich, dass ein multimodales Therapiekonzept mit dem Ziel, Blutdruck, Lipide und den Blutzucker zu normalisieren, im Prinzip günstige Effekte auf mikro- und makrovaskuläre Komplikationen bei diesen Hochrisikopatienten haben kann. Stellvertretend soll hier an die schon etwas ältere Steno-2-Studie (multifaktorielle Interventionsstudie zur kardiovaskulären Erkrankung bei Patienten mit Typ-2Diabetes, Steno Diabetes Center, Dänemark) erinnert werden [6]. Dass dieses Konzept Früchte trägt, beweist eine große Registerstudie aus den USA [7]. Innerhalb von 2 Dekaden (1990-2010) konnte bei Patienten mit einem Diabetes melitus die Inzidenz an Herzinfarkten um 68\%, an Schlaganfällen um 53\%, an Amputationen um $51 \%$ und an terminalem Nierenversagen um $28 \%$ gesenkt werden, bei gleichzeitiger Reduktion der hyperglykämischen Todesfälle um 64\%. Die Autoren schreiben die überraschend großen Effekte einem verbesserten, integrierten Diabetesmanagement zu. Sie beobachteten aber auch eine Steigerung der absoluten Zahlen an Komplikationen durch die permanente Zunahme an Neuerkrankungen, vorzugsweise Typ-2-Diabetes. Es bleibt daher noch viel zu tun auf dem Gebiet insbesondere der Prävention von metabolischem Syndrom und Typ-2-Diabetes.

Wir wünschen Ihnen neue Erkenntnisse zum Wohle Ihrer Patienten und viel Freude beim Studium der Artikel.

Ihre

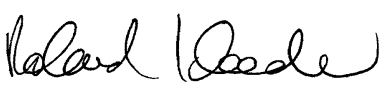

Roland E. Schmieder

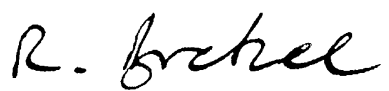

Reinhard G. Bretzel

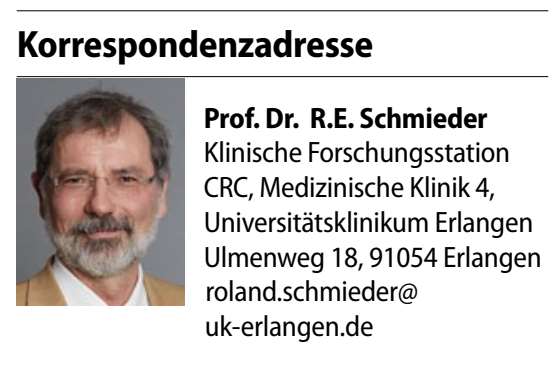

Prof. Dr. med. Dr.
h.c. R.G. Bretzel
Privatpraxis
Wilhelmstraße 12,
35392 Gießen
rgbretzel@googlemail.com

\section{Einhaltung ethischer Richtlinien}

Interessenkonflikt. R.E. Schmieder und R.G. Bretzel geben an, dass kein Interessenkonflikt besteht.

Dieser Beitrag beinhaltet keine Studien an Menschen oderTieren.

\section{Literatur}

1. Lim SS, Vos T, Flaxman AD et al (2012) A comparative risk assessment of burden of disease and injury attributable to 67 risk factors and risk factor clusters in 21 regions, 1990-2010: a systematic analysis for the global burden of disease study 2010. Lancet 380:2224-2260

2. Lewington S, Clarke R, Qizilbash N et al (2002) Age-specific relevance of usual blood pressure to vascular mortality: a meta-analysis of individual data for one million adults in 61 prospective studies. Lancet 360:1903-1913

3. Braun D, Unger H, Wagner A et al (1994) Prevalence of diabetic retinopathy, nephropathy and arterial hypertension in relation to the duration of diabetes mellitus. Diab Stoffw 3:333-338

4. Parving $\mathrm{HH}$, Hommel E, Mathiesen E et al (1988) Prevalence of microalbuminuria, arterial hypertension, retinopathy and neuropathy in patients with insulin dependent diabetes. BMJ 296:156-160
5. Löwel H, Stieber J, Koenig W et al (1999) Das diabetesbedingte Herzinfarktrisiko in einer süddeutschen Bevölkerung: Ergebnisse der MONICA-Augsburg Studien 1985-1994. Diab Stoffw 8:11-21

6. Gaede P, Vedel P, Larsen N et al (2003) Multifactorial intervention and cardiovascular disease in patients with type 2 diabetes. N Engl J Med 348:383393

7. Gregg EW, Li Y, Wang J et al (2014) Changes in diabetes-related complications in the United States, 1990-2010. N Engl J Med 370:1514-1523 